Accepted for publication by the Applied Probability

Trust in Journal of Applied Probability 56.4

\title{
ON MAXIMA OF STATIONARY FIELDS
}

\author{
N. SOJA-KUKIEŁA, ${ }^{*}$ Nicolaus Copernicus University in Toruń, Poland
} 【

\begin{abstract}
Let $\left\{X_{\mathbf{n}}: \mathbf{n} \in \mathbb{Z}^{d}\right\}$ be a weakly dependent stationary random field with maxima $M_{A}:=\sup \left\{X_{\mathbf{i}}: \mathbf{i} \in A\right\}$ for finite $A \subset \mathbb{Z}^{d}$ and $M_{\mathbf{n}}:=\sup \left\{X_{\mathbf{i}}: \mathbf{1} \leq \mathbf{i} \leq \mathbf{n}\right\}$ for $\mathbf{n} \in \mathbb{N}^{d}$. In a general setting we prove that $\mathbb{P}\left(M_{\left(N_{1}(n), N_{2}(n), \ldots, N_{d}(n)\right)} \leq v_{n}\right)$ $=\exp \left(-n^{d} \mathbb{P}\left(X_{\mathbf{0}}>v_{n}, M_{A_{n}} \leq v_{n}\right)\right)+o(1)$ for some increasing sequence of sets $A_{n}$ of size $o\left(n^{d}\right)$, where $\left(N_{1}(n), N_{2}(n), \ldots, N_{d}(n)\right) \rightarrow(\infty, \infty, \ldots, \infty)$ and $N_{1}(n) N_{2}(n) \cdots N_{d}(n) \sim n^{d}$. The sets $A_{n}$ are determined by a translation invariant total order $\preccurlyeq$ on $\mathbb{Z}^{d}$. For a class of fields satisfying a local mixing condition, including $m$-dependent ones, the main theorem holds with a constant finite $A$ replacing $A_{n}$. The above results lead to new formulas for the extremal index for random fields. The new method of calculating limiting probabilities for maxima is compared with some known results and applied to the moving maximum field.
\end{abstract}

Keywords: stationary random fields; extremes; limit theorems; extremal index; $m$-dependence; moving maxima

2010 Mathematics Subject Classification: Primary 60G70

Secondary 60G60

\section{Introduction}

Let us consider a $d$-dimensional stationary random field $\left\{X_{\mathbf{n}}: \mathbf{n} \in \mathbb{Z}^{d}\right\}$ with its partial maxima

$$
M_{A}:=\sup \left\{X_{\mathbf{i}}: \mathbf{i} \in A\right\}
$$

defined for finite $A \subset \mathbb{Z}^{d}$. We also put $M_{\mathbf{j}, \mathbf{n}}:=\sup \left\{X_{\mathbf{i}}: \mathbf{j} \leq \mathbf{i} \leq \mathbf{n}\right\}$ and $M_{\mathbf{n}}:=M_{\mathbf{1}, \mathbf{n}}$ for $\mathbf{j}, \mathbf{n} \in \mathbb{Z}^{d}$. The goal is to study the asymptotic behaviour of $\mathbb{P}\left(M_{\mathbf{N}(n)} \leq v_{n}\right)$ as $n \rightarrow \infty$, for $\left\{v_{n}\right\} \subset \mathbb{R}$ and $\mathbf{N}(n) \rightarrow \infty$ coordinatewise.

In the case $d=1$, when $\left\{X_{n}: n \in \mathbb{Z}\right\}$ is a stationary sequence, the well known result of O'Brien [17, Theorem 2.1] states that under a broad class of circumstances

$$
\mathbb{P}\left(M_{n} \leq v_{n}\right)=\exp \left(-n \mathbb{P}\left(X_{0}>v_{n}, M_{p(n)} \leq v_{n}\right)\right)+o(1)
$$

holds for some $p(n) \rightarrow \infty$ satisfying $p(n)=o(n)$. For $m$-dependent $\left\{X_{n}\right\}$ we can set $p(n):=m$ in formula (11), as Newell [16] shows. It follows that the extremal index $\theta$ for $\left\{X_{n}\right\}$, defined by Leadbetter [14, equals

$$
\theta=\lim _{n \rightarrow \infty} \mathbb{P}\left(M_{p(n)} \leq v_{n} \mid X_{0}>v_{n}\right),
$$

where $p(n)=m$ in the $m$-dependent case. More generally, we can put $p(n)=m$ in (2) whenever Condition $D^{(m+1)}\left(v_{n}\right)$, introduced by Chernick et al. [5], is satisfied.

* Postal address: Faculty of Mathematics and Computer Science, Nicolaus Copernicus University, ul. Chopina 12/18, 87-100 Toruń, Poland; e-mail address: natas@mat.umk.pl 
We recall that the extremal index $\theta \in[0,1]$ is interpreted as the reciprocal of the mean number of high threshold exceedances in a cluster. Formula (2) for $\theta$ may be treated as an answer to the question: Asymptotically, what is the probability that a given element of a cluster of large values is its last element on the right?

Looking for formulas analogous to (11) and (2) for arbitrary $d \in \mathbb{N}_{+}$, one can try to answer the properly formulated $d$-dimensional version of the above question. This point is realized in Sections 3 and 4 . In Section 3 we prove the main result, Theorem 3.1 We establish that in a general setting the approximation

$$
\mathbb{P}\left(M_{\mathbf{N}(n)} \leq v_{n}\right)=\exp \left(-n^{d} \mathbb{P}\left(X_{\mathbf{0}}>v_{n}, M_{A(\mathbf{p}(n))} \leq v_{n}\right)\right)+o(1)
$$

holds with $A(\mathbf{p}(n)) \subset\left\{\mathbf{j} \in \mathbb{Z}^{d}:-\mathbf{p}(n) \leq \mathbf{j} \leq \mathbf{p}(n)\right\}$ defined by (9), $\mathbf{N}(n)$ fulfilling (4) and $\mathbf{p}(n) \rightarrow \infty$ satisfying $\mathbf{p}(n)=o(\mathbf{N}(n))$ and some other rate of growth conditions. For $d=1$ we have $A(p(n))=\{1,2, \ldots, p(n)\}$ and formula (3) simplifies to (11). Corollary 3.3 provides the local mixing condition (16) equivalent to the fact that (3) holds with $\mathbf{p}(n):=(m, m, \ldots, m)$. Section 4 is devoted to considerations concerning the notion of the extremal index for random fields. Formula (18), being a generalization of (2), and its simplified version (19) for fields fulfilling (16) are proposed there. In Section 6 the results from Sections 3 and 4 are applied to describe the asymptotics of partial maxima for the moving maximum field.

In Section 5 we focus on $m$-dependent fields and present a corollary of the main theorem generalizing the aforementioned Newell's [16] formula. We also compare the obtained result with the limit theorem for $m$-dependent fields proven by Jakubowski and Soja-Kukieła [12, Theorem 2.1].

The present paper provides a $d$-dimensional generalization of O'Brien's formula (11) with a handy and immediate conclusion for $m$-dependent fields. Another general theorem by Turkman [19, Theorem 1] is not well applicable in the $m$-dependent case. A recent result obtained independently by Ling [15, Lemma 3.2] is a special case of Theorem 3.1. Other theorems on the topic were given for some subclasses of weakly dependent fields: in the 2-dimensional Gaussian setting by French and Davis [9]; for 2-dimensional moving maxima and moving averages by Basrak and Tafro [3]; for $m$-dependent and max-m-approximable fields by Jakubowski and Soja-Kukieła [12]; for regularly varying fields by $\mathrm{Wu}$ and Samorodnitsky [20]. The proof of Theorem 3.1 presented in the paper, although achieved independently, is similar to proofs of 9 , Lemma 4] and [15, Lemma 3.2].

\section{Preliminaries}

An element $\mathbf{n} \in \mathbb{Z}^{d}$ is often denoted by $\left(n_{1}, n_{2}, \ldots, n_{d}\right)$ and $\|\mathbf{n}\|$ is its sup norm. We write $\mathbf{i} \leq \mathbf{j}$ and $\mathbf{n} \rightarrow \infty$, whenever $i_{l} \leq j_{l}$ and $n_{l} \rightarrow \infty$, respectively, for all $l \in\{1,2, \ldots, d\}$. We put $\mathbf{0}:=(0,0, \ldots, 0), \mathbf{1}:=(1,1, \ldots, 1)$ and $\infty:=(\infty, \infty, \ldots, \infty)$.

In our considerations $\left\{X_{\mathbf{n}}: \mathbf{n} \in \mathbb{Z}^{d}\right\}$ is a $d$-dimensional stationary random field. We ask for the asymptotics of $\mathbb{P}\left(M_{\mathbf{N}(n)} \leq v_{n}\right)$ as $n \rightarrow \infty$, for $\mathbf{N}=\{\mathbf{N}(n): n \in \mathbb{N}\} \subset \mathbb{N}^{d}$ such that

$$
\mathbf{N}(n) \rightarrow \infty \text { and } N^{*}(n):=N_{1}(n) N_{2}(n) \cdots N_{d}(n) \sim n^{d}
$$

and $\left\{v_{n}: n \in \mathbb{N}\right\} \subset \mathbb{R}$.

We are interested in weakly dependent fields. In the paper we assume that

$$
\mathbb{P}\left(M_{\mathbf{N}(n)} \leq v_{n}\right)=\mathbb{P}\left(M_{\mathbf{p}(n)} \leq v_{n}\right)^{k_{n}^{d}}+o(1)
$$


is satisfied for some $r_{n} \rightarrow \infty$ and all $k_{n} \rightarrow \infty$ such that $k_{n}=o\left(r_{n}\right)$, with

$$
\mathbf{p}(n):=\left(\left\lfloor N_{1}(n) / k_{n}\right\rfloor,\left\lfloor N_{2}(n) / k_{n}\right\rfloor, \ldots,\left\lfloor N_{d}(n) / k_{n}\right\rfloor\right) .
$$

Applying the classical fact (see, e.g., O'Brien [17]):

$$
\left(a_{n}\right)^{n}-\exp \left(-n\left(1-a_{n}\right)\right) \rightarrow 0 \quad \text { as } \quad n \rightarrow \infty, \quad \text { for } \quad a_{n} \in[0,1]
$$

we get that (5) implies

$$
\mathbb{P}\left(M_{\mathbf{N}(n)} \leq v_{n}\right)=\exp \left(-k_{n}^{d} \mathbb{P}\left(M_{\mathbf{p}(n)}>v_{n}\right)\right)+o(1) .
$$

Above, $p_{l}(n)=o\left(N_{l}(n)\right)$ for $l \in\{1,2, \ldots, d\}$, which we briefly denote $\mathbf{p}(n)=o(\mathbf{N}(n))$.

Remark 2.1. For $d=1$ weak dependence in the sense of (5) is ensured by any of the following conditions: Leadbetter's $D\left(v_{n}\right)$, O'Brien's AIM $\left(v_{n}\right)$ or Jakubowski's $B_{1}\left(v_{n}\right)$; see [14, 17, 10]. For $d \in \mathbb{N}_{+}$the considered property follows for example from Condition $B_{1}^{\mathbf{N}}\left(v_{n}\right)$ introduced by Jakubowski and Soja-Kukieła [13]. In particular, $m$-dependent fields are weakly dependent; see Section 5. A similar notion of weak dependence was investigated by Ling [15, Lemma 3.1].

Let $\preccurlyeq$ be an arbitrary total order on $\mathbb{Z}^{d}$ which is translation invariant, i.e. $\mathbf{i} \preccurlyeq \mathbf{j}$ implies $\mathbf{i}+\mathbf{k} \preccurlyeq \mathbf{j}+\mathbf{k}$. An example of such an order is the lexicographic order:

$$
\mathbf{i} \preccurlyeq \mathbf{j} \quad \text { iff } \quad\left(\mathbf{i}=\mathbf{j} \quad \text { or } \quad i_{l}<j_{l} \text { for the first } l \text { where } i_{l} \text { and } j_{l} \text { differ }\right) .
$$

We will write $\mathbf{i} \prec \mathbf{j}$ whenever $\mathbf{i} \preccurlyeq \mathbf{j}$ and $\mathbf{i} \neq \mathbf{j}$. For technical needs of further sections, we define the set $A(\mathbf{p}) \subset \mathbb{Z}^{d}$ for each $\mathbf{p} \in \mathbb{N}^{d}$ as follows:

$$
A(\mathbf{p}):=\left\{\mathbf{j} \in \mathbb{Z}^{d}:-\mathbf{p} \leq \mathbf{j} \leq \mathbf{p} \text { and } \mathbf{0} \prec \mathbf{j}\right\} .
$$

\section{Main theorem}

In the following the main result of the paper is presented. The asymptotic behaviour

of $\mathbb{P}\left(M_{\mathbf{N}(n)} \leq v_{n}\right)$ as $n \rightarrow \infty$, for weakly dependent $\left\{X_{\mathbf{n}}\right\}$ and for $\{\mathbf{N}(n)\}$ and $\left\{v_{n}\right\}$ as in Section 2, is described.

Theorem 3.1. Let $\left\{X_{\mathbf{n}}\right\}$ satisfy (5) for some $r_{n} \rightarrow \infty$ and all $k_{n} \rightarrow \infty$ such that $k_{n}=o\left(r_{n}\right)$. If

$$
\liminf _{n \rightarrow \infty} \mathbb{P}\left(M_{\mathbf{N}(n)} \leq v_{n}\right)>0,
$$

then for every $\left\{k_{n}\right\}$ as above

$$
\mathbb{P}\left(M_{\mathbf{N}(n)} \leq v_{n}\right)=\exp \left(-n^{d} \mathbb{P}\left(X_{\mathbf{0}}>v_{n}, M_{A(\mathbf{p}(n))} \leq v_{n}\right)\right)+o(1)
$$

holds with $\mathbf{p}(n)$ and $A(\mathbf{p}(n))$ given by (6) and (9), respectively.

Remark 3.2. If (5) holds for some $k_{n} \rightarrow \infty$, then (10) is implied by the condition

$$
\limsup _{n \rightarrow \infty} n^{d} \mathbb{P}\left(X_{\mathbf{0}}>v_{n}\right)<\infty .
$$

This follows from (8) and the inequality

$$
k_{n}^{d} \mathbb{P}\left(M_{\mathbf{p}(n)}>v_{n}\right) \leq N^{*}(n) \mathbb{P}\left(X_{\mathbf{0}}>v_{n}\right) \sim n^{d} \mathbb{P}\left(X_{\mathbf{0}}>v_{n}\right) .
$$


The proof of the theorem generalizes the reasoning proposed by O'Brien [17, Theorem 2.1] for sequences. A way of dividing the event $\left\{M_{\mathbf{p}(n)}>v_{n}\right\}$ into $p^{*}(n):=$ $p_{1}(n) p_{2}(n) \cdots p_{d}(n)$ mutually exclusive events determined by $\preccurlyeq$ (which are similar in some sense) plays a key role in the proof. An analogous technique was used by French and Davis [9, Lemma 4] in the 2-dimensional Gaussian case. Recently, Ling [15. Lemma 3.1] expanded their result to some non-Gaussian fields. In both papers the authors restrict themselves to the lexicographic order on $\mathbb{Z}^{2}$.

Proof of Theorem 3.1, Let the assumptions of the theorem be satisfied. Then (8) holds. Dividing the set $\left\{M_{\mathbf{p}(n)}>v_{n}\right\}$ into $p^{*}(n)=p_{1}(n) p_{2}(n) \cdots p_{d}(n)$ disjoint sets and applying monotonicity and stationarity we obtain that

$$
\begin{aligned}
\mathbb{P} & \left(M_{\mathbf{p}(n)}>v_{n}\right) \\
& =\sum_{\mathbf{1} \leq \mathbf{j} \leq \mathbf{p}(n)} \mathbb{P}\left(X_{\mathbf{j}}>v_{n}, X_{\mathbf{i}} \leq v_{n} \text { for all } \mathbf{i} \succ \mathbf{j} \text { such that } \mathbf{1} \leq \mathbf{i} \leq \mathbf{p}(n)\right) \\
& \geq \sum_{\mathbf{1} \leq \mathbf{j} \leq \mathbf{p}(n)} \mathbb{P}\left(X_{\mathbf{j}}>v_{n}, X_{\mathbf{i}} \leq v_{n} \text { for all } \mathbf{i} \in A(\mathbf{p}(n))+\mathbf{j}\right) \\
& =p^{*}(n) \mathbb{P}\left(X_{\mathbf{0}}>v_{n}, M_{A(\mathbf{p}(n))} \leq v_{n}\right),
\end{aligned}
$$

which combined with (8) and the fact that $k_{n}^{d} p^{*}(n) \sim n^{d}$ gives

$$
\mathbb{P}\left(M_{\mathbf{N}(n)} \leq v_{n}\right) \leq \exp \left(-n^{d} \mathbb{P}\left(X_{\mathbf{0}}>v_{n}, M_{A(\mathbf{p}(n))} \leq v_{n}\right)\right)+o(1) .
$$

In the second step of the proof we shall show that the inequality reverse to (13) also holds. It is sufficient to consider the case $\mathbb{P}\left(M_{\mathbf{N}(n)} \leq v_{n}\right) \rightarrow \gamma$ for $\gamma \in[0,1]$, and we do so. Since $\gamma=0$ is excluded by assumption (10) and for $\gamma=1$ the proven inequality is obvious, we focus on $\gamma \in(0,1)$. Let us choose $\left\{t_{n}\right\} \subset \mathbb{N}_{+}$so that $t_{n} \rightarrow \infty$ and $t_{n}=o\left(k_{n}\right)$. Put $\mathbf{s}(n):=\left(\left\lfloor N_{1}(n) / t_{n}\right\rfloor,\left\lfloor N_{2}(n) / t_{n}\right\rfloor, \ldots,\left\lfloor N_{d}(n) / t_{n}\right\rfloor\right)$ and $s^{*}(n):=$ $s_{1}(n) s_{2}(n) \cdots s_{d}(n)$. Since $t_{n}=o\left(r_{n}\right)$, (8) holds with $k_{n}$ replaced by $t_{n}$ and $\mathbf{p}(n)$ replaced by $\mathbf{s}(n)$. Also $\mathbf{p}(n)=o(\mathbf{s}(n))$ and $\mathbf{s}(n)=o(\mathbf{N}(n))$. Moreover, for the sets

$$
C(\mathbf{p}(n), \mathbf{s}(n)):=\left\{\mathbf{j} \in \mathbb{Z}^{d}: \mathbf{p}(n)+\mathbf{1} \leq \mathbf{j} \leq \mathbf{s}(n)-\mathbf{p}(n)\right\}
$$

and

$$
B(\mathbf{p}(n), \mathbf{s}(n)):=\left\{\mathbf{j} \in \mathbb{Z}^{d}: \mathbf{1} \leq \mathbf{j} \leq \mathbf{s}(n)\right\} \backslash C(\mathbf{p}(n), \mathbf{s}(n))
$$

we obtain that

$$
\begin{aligned}
\frac{\mathbb{P}\left(M_{\mathbf{s}(n)}>v_{n}, M_{B(\mathbf{p}(n), \mathbf{s}(n))} \leq v_{n}\right)}{\mathbb{P}\left(M_{B(\mathbf{p}(n), \mathbf{s}(n))}>v_{n}\right)} & =\frac{\mathbb{P}\left(M_{\mathbf{s}(n)}>v_{n}\right)-\mathbb{P}\left(M_{B(\mathbf{p}(n), \mathbf{s}(n))}>v_{n}\right)}{\mathbb{P}\left(M_{B(\mathbf{p}(n), \mathbf{s}(n))}>v_{n}\right)} \\
& =\frac{\mathbb{P}\left(M_{\mathbf{s}(n)}>v_{n}\right)}{\mathbb{P}\left(M_{B(\mathbf{p}(n), \mathbf{s}(n))}>v_{n}\right)}-1 \\
& =\frac{\mathbb{P}\left(M_{\mathbf{s}(n)}>v_{n}\right)}{o\left(s^{*}(n) / p^{*}(n)\right) \cdot \mathbb{P}\left(M_{\mathbf{p}(n)}>v_{n}\right)}-1 \\
& =\frac{1+o(1)}{o(1)} \cdot \frac{t_{n}^{d} \mathbb{P}\left(M_{\mathbf{s}(n)}>v_{n}\right)}{k_{n}^{d} \mathbb{P}\left(M_{\mathbf{p}(n)}>v_{n}\right)}-1 .
\end{aligned}
$$


Applying (8) twice, we get

$$
\frac{t_{n}^{d} \mathbb{P}\left(M_{\mathbf{s}(n)}>v_{n}\right)}{k_{n}^{d} \mathbb{P}\left(M_{\mathbf{p}(n)}>v_{n}\right)} \rightarrow \frac{-\log \gamma}{-\log \gamma}=1, \quad \text { as } \quad n \rightarrow \infty,
$$

and consequently

$$
\frac{\mathbb{P}\left(M_{B(\mathbf{p}(n), \mathbf{s}(n))}>v_{n}\right)}{\mathbb{P}\left(M_{\mathbf{s}(n)}>v_{n}, M_{B(\mathbf{p}(n), \mathbf{s}(n))} \leq v_{n}\right)} \rightarrow 0, \quad \text { as } \quad n \rightarrow \infty .
$$

Now, observe that

$$
\begin{aligned}
\mathbb{P}\left(M_{\mathbf{s}(n)}>v_{n}\right) & =\mathbb{P}\left(M_{\mathbf{s}(n)}>v_{n}, M_{B(\mathbf{p}(n), \mathbf{s}(n))} \leq v_{n}\right)+\mathbb{P}\left(M_{B(\mathbf{p}(n), \mathbf{s}(n))}>v_{n}\right) \\
& =\mathbb{P}\left(M_{\mathbf{s}(n)}>v_{n}, M_{B(\mathbf{p}(n), \mathbf{s}(n))} \leq v_{n}\right)(1+o(1)) \\
& \leq \sum_{\mathbf{j} \in C(\mathbf{p}(n), \mathbf{s}(n))} \mathbb{P}\left(X_{\mathbf{j}}>v_{n}, M_{A(\mathbf{p}(n))+\mathbf{j}} \leq v_{n}\right) \cdot(1+o(1)) \\
& \leq s^{*}(n) \mathbb{P}\left(X_{\mathbf{0}}>v_{n}, M_{A(\mathbf{p}(n))} \leq v_{n}\right)(1+o(1)),
\end{aligned}
$$

by property (14), subadditivity and monotonicity of probability, and by stationarity of the field $\left\{X_{\mathbf{n}}\right\}$. Applying (8) with $\left(k_{n}, \mathbf{p}(n)\right)$ replaced by $\left(t_{n}, \mathbf{s}(n)\right)$ and the fact that $t_{n}^{d} s^{*}(n) \sim n^{d}$, we conclude that

$$
\begin{aligned}
\mathbb{P}\left(M_{\mathbf{N}(n)} \leq v_{n}\right) & \geq \exp \left(-t_{n}^{d} s^{*}(n) \mathbb{P}\left(X_{\mathbf{0}}>v_{n}, M_{A(\mathbf{p}(n))} \leq v_{n}\right)(1+o(1))\right)+o(1) \\
& =\exp \left(-n^{d} \mathbb{P}\left(X_{\mathbf{0}}>v_{n}, M_{A(\mathbf{p}(n))} \leq v_{n}\right)\right)+o(1) .
\end{aligned}
$$

Since inequalities (13) and (15) are both satisfied, the proof is complete.

Theorem 3.1immediately yields the following generalization of the result established by Chernick et al. [5, Proposition 1.1] for $d=1$. Assumption (16) given below is a multidimensional counterpart of the local mixing Condition $D^{(m+1)}\left(v_{n}\right)$ defined in [5] for sequences and it is satisfied by, e.g., $m$-dependent fields (see Section 5.1).

Corollary 3.3. Let the assumptions of Theorem 3.1 be satisfied. Then

$$
\mathbb{P}\left(M_{\mathbf{N}(n)} \leq v_{n}\right)=\exp \left(-n^{d} \mathbb{P}\left(X_{\mathbf{0}}>v_{n}, M_{A((m, m, \ldots, m))} \leq v_{n}\right)\right)+o(1)
$$

if and only if

$$
n^{d} \mathbb{P}\left(X_{\mathbf{0}}>v_{n} \geq M_{A((m, m, \ldots, m))}, M_{A(\mathbf{p}(n)) \backslash A((m, m, \ldots, m))}>v_{n}\right) \underset{n \rightarrow \infty}{\longrightarrow} 0,
$$

where $k_{n} \rightarrow \infty$ is such that $k_{n}=o\left(r_{n}\right)$.

We point out that Corollary 3.3 reforms a faulty formula for $m$-dependent fields proposed by Ferreira and Pereira [8, Proposition 2.1]; see [12, Example 5.5]. We also suggest to compare the above condition (16) with assumption $D^{\prime \prime}\left(v_{n}, \mathcal{B}_{n}, \mathcal{V}\right)$ proposed by Pereira et al. [18, Definition 3.1].

Remark 3.4. There exists a close relationship between Theorem 3.1 and compound Poisson approximations in the spirit of Arratia et al. [1, Section 4.2.1]. The random variable

$$
\Lambda_{n}^{(1)}:=\sum_{\mathbf{1} \leq \mathbf{k} \leq \mathbf{N}(n)} \mathbb{1}_{\left\{X_{\mathbf{k}}>v_{n}, M_{\mathbf{k}+A(\mathbf{p}(n))} \leq v_{n}\right\}},
$$


with the expectation $\lambda_{n}^{(1)}:=N^{*}(n) \mathbb{P}\left(X_{\mathbf{0}}>v_{n}, M_{A(\mathbf{p}(n))} \leq v_{n}\right)$, estimates the number of clusters of exceedances over $v_{n}$ in the set $\{\mathbf{k}: \mathbf{1} \leq \mathbf{k} \leq \mathbf{N}(n)\}$ and we have $\mathbb{P}\left(M_{\mathbf{N}(n)} \leq v_{n}\right)=\exp \left(-\lambda_{n}^{(1)}\right)+o(1)$.

Remark 3.5. It is worth noting that translation invariant linear orders on the set of indices $\mathbb{Z}^{d}$ play a significant role in considerations (by Basrak and Planinić [4], Wu and Samorodnitsky [20]) on the extremes of regularly varying fields.

\section{Extremal index}

In this part we apply the results given in Section 3 to establish formulas (18) and (19) for the extremal index $\theta$ for random fields. We refer to Choi [6] or Jakubowski and Soja-Kukieła 12 for definitions and some considerations on the extremal index in the multidimensional setting.

Here we present a method of calculating the number $\theta \in[0,1]$ satisfying

$$
\mathbb{P}\left(M_{\mathbf{N}(n)} \leq v_{n}\right)-\mathbb{P}\left(X_{\mathbf{0}} \leq v_{n}\right)^{\theta n^{d}} \rightarrow 0 \text { as } n \rightarrow \infty,
$$

whenever such $\theta$ exists. Let us observe that according to (7) we have

$$
\mathbb{P}\left(X_{\mathbf{0}} \leq v_{n}\right)^{n^{d}}=\exp \left(-n^{d} \mathbb{P}\left(X_{\mathbf{0}}>v_{n}\right)\right)+o(1)
$$

and, moreover, Theorem 3.1 yields

$$
\mathbb{P}\left(M_{\mathbf{N}(n)} \leq v_{n}\right)=\exp \left(-n^{d} \mathbb{P}\left(X_{\mathbf{0}}>v_{n}, M_{A(\mathbf{p}(n))} \leq v_{n}\right)\right)+o(1)
$$

for $\mathbf{N}(n), v_{n}$ and $\mathbf{p}(n)$ satisfying appropriate assumptions. Hence, provided that

$$
0<\liminf _{n \rightarrow \infty} n^{d} \mathbb{P}\left(X_{\mathbf{0}}>v_{n}\right) \leq \limsup _{n \rightarrow \infty} n^{d} \mathbb{P}\left(X_{\mathbf{0}}>v_{n}\right)<\infty,
$$

condition (17) is satisfied if and only if

$$
\theta=\lim _{n \rightarrow \infty} \mathbb{P}\left(M_{A(\mathbf{p}(n))} \leq v_{n} \mid X_{\mathbf{0}}>v_{n}\right) .
$$

Formula (18), allowing computation of extremal indices $\theta$ for random fields, is a multidimensional generalization of (2). In the special case when assumption (16) is satisfied, it is easy to show that formula (18) simplifies to the following one:

$$
\theta=\lim _{n \rightarrow \infty} \mathbb{P}\left(M_{A((m, m, \ldots, m))} \leq v_{n} \mid X_{\mathbf{0}}>v_{n}\right) .
$$

The above formulas are in line with the interpretation of $\theta$ as the reciprocal of the mean number of high threshold exceedances in a cluster. Indeed, they answer the question: What is the asymptotic probability that a given element of a cluster is the distinguished element of the cluster?, where the distinguished element in a cluster is the greatest one with respect to the order $\preccurlyeq$. Such identification of a unique representative for each cluster is called declustering, declumping or anchoring and has much in common with compound Poisson approximations (see, e.g., 1, 2, 4]).

Remark 4.1. Formula (18) justifies the following definition of the runs estimator $\hat{\theta}_{\mathbf{N}(n)}^{R}$ for the extremal index $\theta$ :

$$
\hat{\theta}_{\mathbf{N}(n)}^{R}:=S_{n}^{-1} \sum_{\mathbf{1}+\mathbf{p}(n) \leq \mathbf{k} \leq \mathbf{N}(n)-\mathbf{p}(n)} \mathbb{1}_{\left\{X_{\mathbf{k}}>v_{n}, M_{\mathbf{k}+A(\mathbf{p}(n))} \leq v_{n}\right\}},
$$

where $S_{n}$ is the number of exceendances over $v_{n}$ in the set $\left\{\mathbf{k} \in \mathbb{Z}^{d}: \mathbf{1} \leq \mathbf{k} \leq \mathbf{N}(n)\right\}$. 


\section{Maxima of $\boldsymbol{m}$-dependent fields}

In this section we focus on $m$-dependent fields. We recall that $\left\{X_{\mathbf{n}}\right\}$ is $m$-dependent for some $m \in \mathbb{N}$, if the families $\left\{X_{\mathbf{i}}: \mathbf{i} \in U\right\}$ and $\left\{X_{\mathbf{j}}: \mathbf{j} \in V\right\}$ are independent for all pairs of finite sets $U, V \subset \mathbb{Z}^{d}$ satisfying $\min \{\|\mathbf{i}-\mathbf{j}\|: \mathbf{i} \in U, \mathbf{j} \in V\}>m$.

Let us assume that $\left\{X_{\mathbf{n}}\right\}$ is $m$-dependent and satisfies (10) for some sequence $\left\{v_{n}\right\} \subset \mathbb{R}$. Then it is easy to show that condition (12) holds too (see [12, Remark 4.2]). Below, we present two methods that can be applied to calculate the limit of $\mathbb{P}\left(M_{\mathbf{N}(n)} \leq v_{n}\right)$. A direct connection between them can be given and we illustrate it in the case $d=2$.

\subsection{First method}

The first of the methods is a consequence of the main result presented in the paper. Since the field $\left\{X_{\mathbf{n}}\right\}$ is $m$-dependent, it satisfies (5D) for each $k_{n} \rightarrow \infty$ such that $k_{n}=$ $o\left(r_{n}\right)$, for some $r_{n} \rightarrow \infty$ (see, e.g., 12]). Moreover, the inequality

$$
\begin{aligned}
& n^{d} \mathbb{P}\left(X_{\mathbf{0}}>v_{n} \geq M_{A((m, m, \ldots, m))}, M_{A(\mathbf{p}(n)) \backslash A((m, m, \ldots, m))}>v_{n}\right) \\
& \quad \leq n^{d} \sum_{\mathbf{i} \in A(\mathbf{p}(n)),\|\mathbf{i}\|>m} \mathbb{P}\left(X_{\mathbf{0}}>v_{n}, X_{\mathbf{i}}>v_{n}\right) \\
& \quad=n^{d} \sum_{\mathbf{i} \in A(\mathbf{p}(n)),\|\mathbf{i}\|>m} \mathbb{P}\left(X_{\mathbf{0}}>v_{n}\right) \mathbb{P}\left(X_{\mathbf{i}}>v_{n}\right) \leq n^{d} \cdot \frac{n^{d}}{k_{n}^{d}} \cdot \mathbb{P}\left(X_{\mathbf{0}}>v_{n}\right)^{2}(1+o(1))
\end{aligned}
$$

holds with the right-hand side tending to zero by (12). From Corollary 3.3 we obtain that

$$
\mathbb{P}\left(M_{\mathbf{N}(n)} \leq v_{n}\right)=\exp \left(-n^{d} \mathbb{P}\left(X_{\mathbf{0}}>v_{n}, M_{A((m, m, \ldots, m))} \leq v_{n}\right)+o(1) .\right.
$$

\subsection{Second method}

The second formula comes from Jakubowski and Soja-Kukieła [12, Theorem 2.1]. It states that we have

$$
\mathbb{P}\left(M_{\mathbf{N}(n)} \leq v_{n}\right)=\exp \left(-n^{d} \sum_{\boldsymbol{\varepsilon} \in\{0,1\}^{d}}(-1)^{\varepsilon_{1}+\varepsilon_{2}+\ldots+\varepsilon_{d}} \mathbb{P}\left(M_{\boldsymbol{\varepsilon},(m, m, \ldots, m)}>v_{n}\right)\right)+o(1)
$$

under the above assumptions on $\left\{X_{\mathbf{n}}\right\}$. This result is a consequence of the Bonferronitype inequality from Jakubowski and Rosiński [11, Theorem 2.1].

\subsection{Comparison}

For $d=1$ both of the formulas simplify to the well-known result of Newell [16:

$$
\mathbb{P}\left(M_{n} \leq v_{n}\right)=\exp \left(-n \mathbb{P}\left(X_{0}>v_{n}, M_{1, m} \leq v_{n}\right)\right)+o(1) .
$$

Each of them allows us to describe the asymptotic behaviour of maxima on the base of tail properties of joint distribution of a fixed finite dimension. To apply the first method, one uses the distribution of the $\left(1+\left((2 m+1)^{d}-1\right) / 2\right)$-element family $\left\{X_{\mathbf{n}}: \mathbf{n} \in\{\mathbf{0}\} \cup A((m, m, \ldots, m))\right\}$. To involve the second method, one bases on the distribution of the $(m+1)^{d}$-element family $\left\{X_{\mathbf{n}}: \mathbf{0} \leq \mathbf{n} \leq(m, m, \ldots, m)\right\}$.

Below a link between the two formulas is described in two ways: a more conceptual one, and one that is shorter but perhaps less intuitive. To avoid annoying technicalities, we focus on $d=2$. 
5.3.1. First approach: counting clusters. Our aim is to calculate the number of clusters of exceedances over $v_{n}$ in the window $W:=\left\{\mathbf{k} \in \mathbb{Z}^{2}: \mathbf{1} \leq \mathbf{k} \leq \mathbf{N}(n)\right\}$ in two different ways and obtain, as a consequence, the equivalence of (20) and (21).

Let the random set $J_{n}$ be given as $J_{n}:=\left\{\mathbf{k} \in W: X_{\mathbf{k}}>v_{n}\right\}$ and let $\leftrightarrow$ be the equivalence relation on $J_{n}$ defined as follows:

$$
\mathbf{i} \leftrightarrow \mathbf{j} \quad \text { whenever } \quad \exists_{l \in \mathbb{N}} \exists_{\mathbf{k}_{0}, \mathbf{k}_{1}, \ldots, \mathbf{k}_{l}, \mathbf{k}_{l+1} \in J_{n}, \mathbf{k}_{0}=\mathbf{i}, \mathbf{k}_{l+1}=\mathbf{j}} \max _{0 \leq h \leq l}\left\|\mathbf{k}_{h+1}-\mathbf{k}_{h}\right\| \leq m,
$$

for $\mathbf{i}, \mathbf{j} \in J_{n}$. We define a cluster as an equivalence class of $\leftrightarrow$ and obtain the partition $\mathcal{C}_{n}:=J_{n} / \leftrightarrow$ of $J_{n}$ into $\Lambda_{n}:=\# \mathcal{C}_{n}$ clusters. We put $\lambda_{n}:=\mathbb{E}\left(\Lambda_{n}\right), \mathcal{C}_{n}^{\prime}:=\left\{C \in \mathcal{C}_{n}:\right.$ $\left.\max _{\mathbf{i}, \mathbf{j} \in C}\|\mathbf{i}-\mathbf{j}\| \leq m\right\}, \mathcal{C}_{n}^{\prime \prime}:=\mathcal{C}_{n} \backslash \mathcal{C}_{n}^{\prime}, \Lambda_{n}^{\prime}:=\# \mathcal{C}_{n}^{\prime}$ and $\lambda_{n}^{\prime}:=\mathbb{E}\left(\Lambda_{n}^{\prime}\right)$.

Let $\Lambda_{n}^{(1)}$ and $\lambda_{n}^{(1)}$, associated with the method presented in Section [5.1] be defined as in Remark 3.4 with $\mathbf{p}(n):=(m, m)$. Recall that we have $A(m, m)=\left\{\mathbf{j} \in \mathbb{Z}^{2}\right.$ : $(-m,-m) \leq \mathbf{j} \leq(m, m)$ and $(0,0) \prec \mathbf{j}\}$. Analogously (see [12, Remark 2.2]) we define $\Lambda_{n}^{(2)}$ and $\lambda_{n}^{(2)}$ related with the method from Section 5.2 as follows:

$$
\begin{aligned}
\Lambda_{n}^{(2)} & :=\sum_{\mathbf{k} \in W} \sum_{\boldsymbol{\varepsilon} \in\{0,1\}^{2}}(-1)^{\varepsilon_{1}+\varepsilon_{2}} \mathbb{1}_{\left\{M_{\mathbf{k}+\boldsymbol{\varepsilon}, \mathbf{k}+(m, m)}>v_{n}\right\}}, \\
\lambda_{n}^{(2)} & :=\mathbb{E}\left(\Lambda_{n}^{(2)}\right)=N^{*}(n) \sum_{\boldsymbol{\varepsilon} \in\{0,1\}^{2}}(-1)^{\varepsilon_{1}+\varepsilon_{2}} \mathbb{P}\left(M_{\boldsymbol{\varepsilon},(m, m)}>v_{n}\right) .
\end{aligned}
$$

Assume that $C \in \mathcal{C}_{n}^{\prime}$. Let $B(C, m):=\left\{\mathbf{j} \in \mathbb{Z}^{2}:\|\mathbf{j}-\mathbf{k}\| \leq m\right.$ for some $\left.\mathbf{k} \in C\right\}$ and suppose that $M_{B(C, m) \backslash C} \leq v_{n}$ holds (which obviously is satisfied in the typical case $B(C, m) \subset W)$. Observe that for such $C$ we have

$$
\sum_{\mathbf{k} \in C} \mathbb{1}_{\left\{M_{A(m, m)+\mathbf{k}} \leq v_{n}\right\}}=\sum_{\mathbf{k} \in C} \mathbb{1}_{\{\mathbf{k} \text { is the largest element of } C \text { with respect to } \preccurlyeq\}}=1
$$

and

$$
\sum_{\mathbf{k} \in \bar{C} \boldsymbol{\varepsilon} \in\{0,1\}^{2}}(-1)^{\varepsilon_{1}+\varepsilon_{2}} \mathbb{1}_{\left\{M_{\mathbf{k}+\boldsymbol{\varepsilon}, \mathbf{k}+(m, m)}>v_{n}\right\}}=\mathbb{1}_{\left\{M_{\mathbf{k}(C), \mathbf{k}(C)+(m, m)}>v_{n}\right\}}=1,
$$

where $\bar{C}:=\left\{\mathbf{k} \in \mathbb{Z}^{2}: \mathbf{k}+\mathbf{i} \in C\right.$ for some $\left.\mathbf{0} \leq \mathbf{i} \leq(m, m)\right\}$ and $\mathbf{k}(C)$ satisfies the condition $C \subset\left\{\mathbf{k} \in \mathbb{Z}^{2}: \mathbf{k}(C) \leq \mathbf{k} \leq \mathbf{k}(C)+(m, m)\right\}$.

We will show that each of the following equalities holds:

$$
\begin{aligned}
\delta_{n} & :=\mathbb{E}\left|\Lambda_{n}-\Lambda_{n}^{\prime}\right|=\mathbb{E}\left(\Lambda_{n}-\Lambda_{n}^{\prime}\right)=o(1) ; \\
\delta_{n}^{(1)} & :=\mathbb{E}\left|\Lambda_{n}^{(1)}-\Lambda_{n}^{\prime}\right|=o(1) ; \\
\delta_{n}^{(2)} & :=\mathbb{E}\left|\Lambda_{n}^{(2)}-\Lambda_{n}^{\prime}\right|=o(1) .
\end{aligned}
$$

This will entail the condition $\lambda_{n}=\lambda_{n}^{\prime}+o(1)=\lambda_{n}^{(1)}+o(1)=\lambda_{n}^{(2)}+o(1)$ and complete this section.

To show (24), observe that the event $\left\{\# \mathcal{C}_{n}^{\prime \prime}=l\right\}$, for $l \in \mathbb{N}_{+}$, implies that there exist pairs $\mathbf{j}(i, a), \mathbf{j}(i, b) \in J_{n}$, for $i \in\{1,2, \ldots, l\}$, such that $m<\|\mathbf{j}(i, a)-\mathbf{j}(i, b)\| \leq 2 m$ holds for each $i$ and $\left\|\mathbf{j}\left(i_{1}, c_{1}\right)-\mathbf{j}\left(i_{2}, c_{2}\right)\right\|>m$ is satisfied for $i_{1} \neq i_{2}$ and $c_{1}, c_{2} \in\{a, b\}$. Thus we have

$$
\delta_{n}=\sum_{l=1}^{\infty} l \mathbb{P}\left(\# \mathcal{C}_{n}^{\prime \prime}=l\right) \leq \sum_{l=1}^{\infty} l\left(N^{*}(n)\left((4 m+1)^{2}-(2 m+1)^{2}\right) \mathbb{P}\left(X_{\mathbf{0}}>v_{n}\right)^{2}\right)^{l} .
$$


Since $q_{n}:=N^{*}(n)\left((4 m+1)^{2}-(2 m+1)^{2}\right) \mathbb{P}\left(X_{\mathbf{0}}>v_{n}\right)^{2}=o(1)$ by (12), we obtain that $\delta_{n} \leq \sum_{l=1}^{\infty} l\left(q_{n}\right)^{l}=q_{n}\left(1-q_{n}\right)^{-2}$ for all large $n$ 's and finally $\delta_{n}=o(1)$.

Before we establish (25) and (26), we will give an upper bound for the probability that a fixed $\mathbf{k} \in W$ belongs to a large cluster. Note that we have

$$
\begin{aligned}
\mathbb{P}(\mathbf{k} \in & \left.C \text { for some } C \in \mathcal{C}_{n}^{\prime \prime}\right) \\
= & \mathbb{P}\left(\mathbf{k} \in C \text { and }\|\mathbf{j}-\mathbf{k}\| \leq m \text { for all } \mathbf{j} \in C, \text { for some } C \in \mathcal{C}_{n}^{\prime \prime}\right) \\
& +\mathbb{P}\left(\mathbf{k} \in C \text { and }\|\mathbf{j}-\mathbf{k}\|>m \text { for some } \mathbf{j} \in C, \text { for some } C \in \mathcal{C}_{n}^{\prime \prime}\right) \\
\leq & \mathbb{P}\left(\|\mathbf{i}-\mathbf{k}\| \leq m \text { and }\|\mathbf{j}-\mathbf{k}\| \leq m \text { and }\|\mathbf{i}-\mathbf{j}\|>m, \text { for some } \mathbf{i}, \mathbf{j} \in J_{n}\right) \\
& +\mathbb{P}\left(\mathbf{k} \in J_{n} \text { and } m<\|\mathbf{k}-\mathbf{j}\| \leq 2 m \text { for some } \mathbf{j} \in J_{n}\right) \\
\leq & \left((2 m+1)^{4}+\left((4 m+1)^{2}-(2 m+1)^{2}\right)\right) \mathbb{P}\left(X_{\mathbf{0}}>v_{n}\right)^{2}=a(m) \mathbb{P}\left(X_{\mathbf{0}}>v_{n}\right)^{2}
\end{aligned}
$$

with $a(m):=(2 m+1)^{4}+4 m(3 m+1)$.

Applying observation (22), property (27) and taking into account estimation errors for clusters situated near the edges of the window $W$, we obtain

$$
\begin{aligned}
\delta_{n}^{(1)} & =\mathbb{E}\left|\sum_{\mathbf{k} \in W}\left(\mathbb{1}_{\left\{\mathbf{k} \in \cup \mathcal{C}_{n}^{\prime}, M_{\mathbf{k}+A(m, m)} \leq v_{n}\right\}}+\mathbb{1}_{\left\{\mathbf{k} \in \bigcup \mathcal{C}_{n}^{\prime \prime}, M_{\mathbf{k}+A(m, m)} \leq v_{n}\right\}}\right)-\Lambda_{n}^{\prime}\right| \\
& \leq \mathbb{E}\left|\sum_{C \in \mathcal{C}_{n}^{\prime}} \sum_{\mathbf{k} \in C} \mathbb{1}_{\left\{M_{\mathbf{k}+A(m, m)} \leq v_{n}\right\}}-\Lambda_{n}^{\prime}\right|+\mathbb{E}\left(\sum_{\mathbf{k} \in W} \mathbb{1}_{\left\{\mathbf{k} \in \bigcup \mathcal{C}_{n}^{\prime \prime}\right\}}\right) \\
& \leq 2 m\left(N_{1}(n)+N_{2}(n)\right) \mathbb{P}\left(X_{\mathbf{0}}>v_{n}\right)+a(m) N^{*}(n) \mathbb{P}\left(X_{\mathbf{0}}>v_{n}\right)^{2},
\end{aligned}
$$

which combined with assumption (12) implies (25). Quite similarly, using (23) instead of (22) and writing $\bar{W}:=\left\{\mathbf{k} \in \mathbb{Z}^{2}: \mathbf{1}-(m, m) \leq \mathbf{k} \leq \mathbf{N}(n)\right\}$, we conclude that

$$
\begin{aligned}
\delta_{n}^{(2)} \leq & \mathbb{E}\left|\sum_{\mathbf{k} \in \bar{W}} \mathbb{1}_{\left\{\mathbf{k} \in \bar{C} \text { for some } C \in \mathcal{C}_{n}^{\prime}\right\}} \sum_{\boldsymbol{\varepsilon} \in\{0,1\}^{2}}(-1)^{\varepsilon_{1}+\varepsilon_{2}} \mathbb{1}_{\left\{M_{\mathbf{k}+\boldsymbol{\varepsilon}, \mathbf{k}+(m, m)}>v_{n}\right\}}-\Lambda_{n}^{\prime}\right| \\
& +\mathbb{E}\left(\sum_{\mathbf{k} \in \bar{W} \backslash W} \sum_{\boldsymbol{\varepsilon} \in\{0,1\}^{2}}\left|(-1)^{\varepsilon_{1}+\varepsilon_{2}} \mathbb{1}_{\left\{M_{\mathbf{k}+\boldsymbol{\varepsilon}, \mathbf{k}+(m, m)}>v_{n}\right\}}\right|\right) \\
& +2 \mathbb{E}\left(\sum_{\mathbf{k} \in W} \mathbb{1}_{\left\{\mathbf{k} \in \bar{C} \text { for some } C \in \mathcal{C}_{n}^{\prime \prime}\right\}}\right) \\
\leq & \mathbb{E}\left|\sum_{C \in \mathcal{C}_{n}^{\prime}} \sum_{\mathbf{k} \in \bar{C} \boldsymbol{\varepsilon} \in\{0,1\}^{2}}(-1)^{\varepsilon_{1}+\varepsilon_{2}} \mathbb{1}_{\left\{M_{\mathbf{k}+\boldsymbol{\varepsilon}, \mathbf{k}+(m, m)}>v_{n}\right\}}-\Lambda_{n}^{\prime}\right| \\
& +\sum_{\mathbf{k} \in \bar{W} \backslash W}(2 m+1) \mathbb{P}\left(X_{\mathbf{0}}>v_{n}\right)+2 N^{*}(n) \mathbb{P}\left(\mathbf{k} \in \bar{C} \text { for some } C \in \mathcal{C}_{n}^{\prime \prime}\right) \\
\leq & 2 m(2 m+1)\left(N_{1}(n)+N_{2}(n)\right) \mathbb{P}\left(X_{\mathbf{0}}>v_{n}\right) \\
& +m(2 m+1)\left(N_{1}(n)+N_{2}(n)+m\right) \mathbb{P}\left(X_{\mathbf{0}}>v_{n}\right)+2(m+1)^{2} a(m) N^{*}(n) \mathbb{P}\left(X_{\mathbf{0}}>v_{n}\right)^{2} .
\end{aligned}
$$

Since the right-hand side tends to zero by (12), property (26) follows. 
5.3.2. Second approach: direct verification. In this part we assume that $\preccurlyeq$ is the lexicographic order on $\mathbb{Z}^{2}$. Let us notice that

$$
\begin{aligned}
\mathbb{P}\left(M_{(0,0),(m, m)}>v_{n}\right)-\mathbb{P}\left(M_{(1,0),(m, m)}>v_{n}\right)-\mathbb{P}\left(M_{(0,1),(m, m)}>v_{n}\right)+\mathbb{P}\left(M_{(1,1),(m, m)}>v_{n}\right) \\
=\mathbb{P}\left(X_{(0,0)}>v_{n}, M_{R((m, m))} \leq v_{n}\right) \\
\quad-\mathbb{P}\left(M_{(0,1),(0, m)}>v_{n}, M_{(1,0),(m, 0)}>v_{n}, M_{(1,1),(m, m)} \leq v_{n}\right)
\end{aligned}
$$

is true with $R\left(\left(p_{1}, p_{2}\right)\right):=A\left(\left(p_{1}, p_{2}\right)\right) \cap \mathbb{N}^{2}$, where on the left-hand side of the equation the sum of probabilities from (21) for $d=2$ appears. Next, let us look at the exponent in (20) and observe that

$$
\begin{aligned}
& \mathbb{P}\left(X_{(0,0)}>v_{n}, M_{A((m, m))} \leq v_{n}\right) \\
& =\mathbb{P}\left(X_{(0,0)}>v_{n}, M_{R((m, m))} \leq v_{n}\right) \\
& \quad-\mathbb{P}\left(X_{(0,0)}>v_{n}, M_{R((m, m))} \leq v_{n}, M_{(1,-m),(m,-1)}>v_{n}\right)
\end{aligned}
$$

holds and, moreover, the second summand of the right-hand side satisfies

$$
\begin{aligned}
\mathbb{P} & \left(X_{(0,0)}>v_{n}, M_{R((m, m))} \leq v_{n}, M_{(1,-m),(m,-1)}>v_{n}\right) \\
& =\sum_{l=1}^{m} \mathbb{P}\left(X_{(0,0)}>v_{n}, M_{R((m, m))} \leq v_{n}, M_{(1,-l),(m,-l)}>v_{n}, M_{(1,-l+1),(m,-1)} \leq v_{n}\right) \\
= & \sum_{l=1}^{m} \mathbb{P}\left(X_{(0,0)}>v_{n}, M_{R((m, m-l))} \leq v_{n}, M_{(1,-l),(m,-l)}>v_{n}, M_{(1,-l+1),(m,-1)} \leq v_{n}\right) \\
= & +o\left(n^{-2}\right) \\
& \sum_{l=1}^{m} \mathbb{P}\left(X_{(0, l)}>v_{n}, M_{(0, l)+R((m, m-l))} \leq v_{n}, M_{(1,0),(m, 0)}>v_{n}, M_{(1,1),(m, l-1)} \leq v_{n}\right) \\
& =\mathbb{P}\left(M_{(0,1),(0, m)}>v_{n}, M_{(1,0),(m, 0)}>v_{n}, M_{(1,1),(m, m)} \leq v_{n}\right)+o\left(n^{-2}\right) .
\end{aligned}
$$

In the above statement probabilities of mutually exclusive events are summed up and $m$-dependence, assumption (12) and stationarity are applied. Finally, we obtain

$$
\begin{array}{r}
\mathbb{P}\left(M_{(0,0),(m, m)}>v_{n}\right)-\mathbb{P}\left(M_{(1,0),(m, m)}>v_{n}\right)-\mathbb{P}\left(M_{(0,1),(m, m)}>v_{n}\right)+\mathbb{P}\left(M_{(1,1),(m, m)}>v_{n}\right) \\
=\mathbb{P}\left(X_{(0,0)}>v_{n}, M_{A((m, m))} \leq v_{n}\right)+o\left(n^{-2}\right) .
\end{array}
$$

Summarizing, we have confirmed that both presented methods lead to the same result.

Remark 5.1. The above reasoning for $m$-dependent fields can also be applied in the general setting. Suppose that formula (3), with $\preccurlyeq$ the lexicographic order on $\mathbb{Z}^{2}$, describes the asymptotics of partial maxima of the stationary field $\left\{X_{\mathbf{n}}: \mathbf{n} \in \mathbb{Z}^{2}\right\}$. Then

$$
\mathbb{P}\left(M_{\mathbf{N}(n)} \leq v_{n}\right)=\exp \left(-n^{2} \sum_{\varepsilon \in\{0,1\}^{2}}(-1)^{\varepsilon_{1}+\varepsilon_{2}} \mathbb{P}\left(M_{\boldsymbol{\varepsilon}, \mathbf{p}(n)}>v_{n}\right)\right)+o(1)
$$


holds if and only if $\left\{X_{\mathbf{n}}\right\}$ satisfies the following condition:

$$
\sum_{l=1}^{p_{2}(n)} \mathbb{P}\left(X_{\mathbf{0}}>v_{n}, M_{U_{l}(\mathbf{p}(n))}>v_{n}, M_{V_{l}(\mathbf{p}(n))}>v_{n}, M_{W_{l}(\mathbf{p}(n))} \leq v_{n}\right)=o\left(n^{-2}\right),
$$

where $U_{l}(\mathbf{p}):=\left\{0, \ldots, p_{1}\right\} \times\left\{p_{2}-l+1, \ldots, p_{2}\right\}, V_{l}(\mathbf{p}):=\left\{1, \ldots, p_{1}\right\} \times\{-l\}$ and $W_{l}(\mathbf{p}):=A(\mathbf{p}) \cap\left(\mathbb{Z} \times\left\{-l+1, \ldots, p_{2}-l\right\}\right)$. Formula (28) generalizes (21). In the present section we have used the fact that $m$-dependent fields satisfy (29) with $\mathbf{p}(n):=(m, m)$.

\section{Example: moving maxima}

Below, we use the results from Sections 3 and 4 to describe the asymptotics of partial maxima for the moving maximum field. We note that approaches to the problem using different methods can be found in Basrak and Tafro [3 or Jakubowski and SojaKukieła [12. In the first paper compound Poisson point process approximation is applied while in the second one the authors combine a Bonferroni-like inequality and max-m-approximability.

In the following $\left\{Z_{\mathbf{n}}\right\}$ is an array of i.i.d. random variables satisfying

$$
\mathbb{P}\left(\left|Z_{\mathbf{0}}\right|>x\right)=x^{-\alpha} L(x),
$$

for some index $\alpha>0$ and slowly varying function $L$, and

$$
\frac{\mathbb{P}\left(Z_{\mathbf{0}}>x\right)}{\mathbb{P}\left(\left|Z_{\mathbf{0}}\right|>x\right)}=p \quad \text { as } \quad x \rightarrow \infty, \quad \text { for some } \quad p \in[0,1] .
$$

We define $a_{n}:=\inf \left\{y>0: \mathbb{P}\left(\left|Z_{\mathbf{0}}\right|>y\right) \leq n^{-d}\right\}$ and $v_{n}:=a_{n} v$ with fixed $v>0$. Then

$$
n^{d} \mathbb{P}\left(\left|Z_{\mathbf{0}}\right|>v_{n}\right) \rightarrow v^{-\alpha} \quad \text { as } n \rightarrow \infty .
$$

Let us consider the moving maximum field $\left\{X_{\mathbf{n}}\right\}$ defined as

$$
X_{\mathbf{n}}=\sup _{\mathbf{j} \in \mathbb{Z}^{d}} c_{\mathbf{j}} Z_{\mathbf{n}+\mathbf{j}}
$$

where $c_{\mathbf{j}} \in \mathbb{R}$, not all equal to zero, satisfy

$$
\sum_{\mathbf{j} \in \mathbb{Z}^{d}}\left|c_{\mathbf{j}}\right|^{\beta}<\infty \text { for some } 0<\beta<\alpha .
$$

From Cline [7, Lemma 2.2] it follows that the field $\left\{X_{\mathbf{n}}\right\}$ is well defined and

$$
\begin{aligned}
\lim _{x \rightarrow \infty} \frac{\mathbb{P}\left(X_{\mathbf{0}}>x\right)}{\mathbb{P}\left(\left|Z_{\mathbf{0}}\right|>x\right)} & =\lim _{x \rightarrow \infty} \frac{\mathbb{P}\left(\sup _{\mathbf{j} \in \mathbb{Z}^{d}} c_{\mathbf{j}} Z_{\mathbf{j}}>x\right)}{\mathbb{P}\left(\left|Z_{\mathbf{0}}\right|>x\right)} \\
& =\lim _{x \rightarrow \infty} \frac{\sum_{\mathbf{j} \in \mathbb{Z}^{d}} \mathbb{P}\left(c_{\mathbf{j}} Z_{\mathbf{j}}>x\right)}{\mathbb{P}\left(\left|Z_{\mathbf{0}}\right|>x\right)}=p \sum_{c_{\mathbf{j}}>0} c_{\mathbf{j}}^{\alpha}+q \sum_{c_{\mathbf{j}}<0}\left|c_{\mathbf{j}}\right|^{\alpha} .
\end{aligned}
$$

with $q:=1-p$.

Since the moving maximum field is max-m-approximable, there exists a phantom distribution function for $\left\{X_{\mathbf{n}}\right\}$ (see Jakubowski and Soja-Kukieła [12]) and hence the 
field is weakly dependent in the sense of (5). We will apply Theorem 3.1 with $\preccurlyeq$ being the lexicographic order on $\mathbb{Z}^{d}$ to describe the asymptotics of partial maxima. Let us observe that the exponent in (11) satisfies

$$
\begin{array}{rl}
n^{d} & \mathbb{P}\left(X_{\mathbf{0}}>v_{n}, M_{A(\mathbf{p}(n))} \leq v_{n}\right) \\
= & n^{d} \mathbb{P}\left(\bigcup_{\mathbf{j} \in \mathbb{Z}^{d}}\left\{c_{\mathbf{j}} Z_{\mathbf{j}}>v_{n}\right\}, \bigcap_{\mathbf{k} \in \mathbb{Z}^{d}}\left\{\max _{\mathbf{i} \in A(\mathbf{p}(n))}\left(c_{\mathbf{k}-\mathbf{i}} Z_{\mathbf{k}}\right) \leq v_{n}\right\}\right) \\
= & n^{d} \sum_{\mathbf{j} \in \mathbb{Z}^{d}} \mathbb{P}\left(c_{\mathbf{j}} Z_{\mathbf{j}}>v_{n}, \bigcap_{\mathbf{k} \in \mathbb{Z}^{d}}\left\{\max _{\mathbf{i} \in A(\mathbf{p}(n))}\left(c_{\mathbf{k}-\mathbf{i}} Z_{\mathbf{k}}\right) \leq v_{n}\right\}\right)+o(1) \\
= & n^{d} \sum_{\mathbf{j} \in \mathbb{Z}^{d}} \mathbb{P}\left(c_{\mathbf{j}} Z_{\mathbf{j}}>v_{n} \geq \max _{\mathbf{i} \in A(\mathbf{p}(n))}\left(c_{\mathbf{j}-\mathbf{i}} Z_{\mathbf{j}}\right), \bigcap_{\mathbf{k} \neq \mathbf{j}}\left\{\max _{\mathbf{i} \in A(\mathbf{p}(n))}\left(c_{\mathbf{k}-\mathbf{i}} Z_{\mathbf{k}}\right) \leq v_{n}\right\}\right)+o(1) \\
= & n^{d} \sum_{\mathbf{j} \in \mathbb{Z}^{d}} \mathbb{P}\left(c_{\mathbf{j}} Z_{\mathbf{j}}>v_{n} \geq \max _{\mathbf{i} \in A(\mathbf{p}(n))}\left(c_{\mathbf{j}-\mathbf{i}} Z_{\mathbf{j}}\right)\right) \mathbb{P}\left(\bigcap_{\mathbf{k} \neq \mathbf{j}}\left\{\max _{\mathbf{i} \in A(\mathbf{p}(n))}\left(c_{\mathbf{k}-\mathbf{i}} Z_{\mathbf{k}}\right) \leq v_{n}\right\}\right)+o(1),
\end{array}
$$

as $n \rightarrow \infty$, where the second equality follows from (33) combined with the choice of $\left\{v_{n}\right\}$ and the last one is a consequence of the independence of $Z_{\mathbf{j}}$ for $\mathbf{j} \in \mathbb{Z}^{d}$. Note that we have

$$
\begin{aligned}
\mathbb{P}\left(\bigcap _ { \mathbf { k } \neq \mathbf { j } } \left\{\max _{\mathbf{i} \in A(\mathbf{p}(n))}\left(c_{\mathbf{k}-\mathbf{i}} Z_{\mathbf{k}}\right)\right.\right. & \left.\left.\leq v_{n}\right\}\right) \geq \mathbb{P}\left(\bigcap_{\mathbf{k} \in \mathbb{Z}^{d}}\left\{\max _{\mathbf{i} \in A(\mathbf{p}(n))}\left(c_{\mathbf{k}-\mathbf{i}} Z_{\mathbf{k}}\right) \leq v_{n}\right\}\right) \\
& \geq \mathbb{P}\left(M_{A(\mathbf{p}(n))} \leq v_{n}\right) \geq 1-o\left(n^{d}\right) \mathbb{P}\left(X_{\mathbf{0}}>v_{n}\right)=1+o(1) .
\end{aligned}
$$

Moreover, for $p_{\min }(n):=\min \left\{p_{l}(n): 1 \leq l \leq d\right\}$ and for $q(n) \in \mathbb{N}$ chosen so that $q(n) \rightarrow \infty, q(n) \leq p_{\min }(n) / 2$ and $q(n)^{d} n^{d} \mathbb{P}\left(\max \left\{c_{\mathbf{i}} Z_{\mathbf{0}}:\|\mathbf{i}\|>p_{\min }(n) / 2\right\}>v_{n}\right) \rightarrow 0$, it follows that

$$
\begin{array}{rl}
\mid n^{d} \sum_{\mathbf{j} \in \mathbb{Z}^{d}} & \mathbb{P}\left(c_{\mathbf{j}} Z_{\mathbf{j}}>v_{n} \geq \max _{\mathbf{i} \in A(\mathbf{p}(n))}\left(c_{\mathbf{j}-\mathbf{i}} Z_{\mathbf{j}}\right)\right)-n^{d} \sum_{\mathbf{j} \in \mathbb{Z}^{d}} \mathbb{P}\left(c_{\mathbf{j}} Z_{\mathbf{j}}>v_{n} \geq \underset{\mathbf{0} \prec \mathbf{i}}{\sup }\left(c_{\mathbf{j}-\mathbf{i}} Z_{\mathbf{j}}\right)\right) \mid \\
& \leq n^{d} \sum_{\mathbf{j} \in \mathbb{Z}^{d}} \mathbb{P}\left(c_{\mathbf{j}} Z_{\mathbf{j}}>v_{n}, \sup _{\mathbf{0} \prec \mathbf{i} \notin A(\mathbf{p}(n))}\left(c_{\mathbf{j}-\mathbf{i}} Z_{\mathbf{j}}\right)>v_{n}\right) \\
& \leq n^{d} \sum_{\mathbf{j} \in \mathbb{Z}^{d}} \mathbb{P}\left(c_{\mathbf{j}} Z_{\mathbf{j}}>v_{n}, \sup _{\|\mathbf{i}\|>p_{\min }(n)}\left(c_{\mathbf{j}-\mathbf{i}} Z_{\mathbf{j}}\right)>v_{n}\right) \\
& \leq n^{d} \sum_{\|\mathbf{j}\| \leq q(n)} \mathbb{P}\left(\sup _{\|\mathbf{i}\|>p_{\min }(n)}\left(c_{\mathbf{j}-\mathbf{i}} Z_{\mathbf{j}}\right)>v_{n}\right)+n^{d} \sum_{\|\mathbf{j}\|>q(n)} \mathbb{P}\left(c_{\mathbf{j}} Z_{\mathbf{j}}>v_{n}\right) \\
\leq & n^{d}(2 q(n)+1)^{d} \mathbb{P}\left(\sup _{\|\mathbf{i}\|>p_{\min }(n) / 2}\left(c_{\mathbf{i}} Z_{\mathbf{0}}\right)>v_{n}\right)+n^{d} \sum_{\|\mathbf{j}\|>q(n)} \mathbb{P}\left(c_{\mathbf{j}} Z_{\mathbf{j}}>v_{n}\right)
\end{array}
$$


The first summand on the right-hand side tends to zero due to the choice of $q(n)$ and the second one tends to zero by properties (32), (33) and the definition of $v_{n}$. We conclude that

$$
\begin{aligned}
n^{d} \mathbb{P}\left(X_{\mathbf{0}}>v_{n},\right. & \left.M_{A(\mathbf{p}(n))} \leq v_{n}\right) \\
= & \left(n^{d} \sum_{\mathbf{j} \in \mathbb{Z}^{d}} \mathbb{P}\left(c_{\mathbf{j}} Z_{\mathbf{j}}>v_{n} \geq \sup _{\mathbf{0} \prec \mathbf{i}}\left(c_{\mathbf{j}-\mathbf{i}} Z_{\mathbf{j}}\right)\right)+o(1)\right)(1+o(1))+o(1) .
\end{aligned}
$$

To complete the above calculation, it is sufficient to observe that

$$
\begin{array}{r}
n^{d} \sum_{\mathbf{j} \in \mathbb{Z}^{d}} \mathbb{P}\left(c_{\mathbf{j}} Z_{\mathbf{j}}>v_{n} \geq \sup _{\mathbf{0} \prec \mathbf{i}}\left(c_{\mathbf{j}-\mathbf{i}} Z_{\mathbf{j}}\right)\right)=n^{d} \sum_{\mathbf{j} \in \mathbb{Z}^{d}} \mathbb{P}\left(c_{\mathbf{j}} Z_{\mathbf{0}}>v_{n} \geq \sup _{\mathbf{i} \prec \mathbf{j}}\left(c_{\mathbf{i}} Z_{\mathbf{0}}\right)\right) \\
=n^{d} \mathbb{P}\left(\sup _{\mathbf{j} \in \mathbb{Z}^{d}}\left(c_{\mathbf{j}} Z_{\mathbf{0}}\right)>v_{n}\right) \rightarrow\left(p\left(c^{+}\right)^{\alpha}+q\left(c^{-}\right)^{\alpha}\right) v^{-\alpha},
\end{array}
$$

with $c^{+}:=\max _{\mathbf{i} \in \mathbb{Z}^{d}} \max \left\{c_{\mathbf{i}}, 0\right\}$ and $c^{-}:=\max _{\mathbf{i} \in \mathbb{Z}^{d}} \max \left\{-c_{\mathbf{i}}, 0\right\}$. By (11) we obtain

$$
\mathbb{P}\left(M_{\mathbf{N}(n)} \leq v_{n}\right) \rightarrow \exp \left(-\left(p\left(c^{+}\right)^{\alpha}+q\left(c^{-}\right)^{\alpha}\right) v^{-\alpha}\right), \quad \text { as } n \rightarrow \infty .
$$

Applying formula (18) and property (33), we calculate the extremal index of $\left\{X_{\mathbf{n}}\right\}$ as follows

$$
\theta=\frac{p\left(c^{+}\right)^{\alpha}+q\left(c^{-}\right)^{\alpha}}{p \sum_{c_{\mathbf{j}}>0} c_{\mathbf{j}}^{\alpha}+q \sum_{c_{\mathbf{j}}<0}\left|c_{\mathbf{j}}\right|^{\alpha}},
$$

whenever the denominator is positive, which is the only interesting case.

\section{Acknowledgements}

The author would like to thank the referees and the associate editor for comments and suggestions which improved the manuscript significantly.

\section{References}

[1] Arratia, R., Goldstein, L. And Gordon, L. (1990). Poisson approximation and the ChenStein method. Statist. Sci. 5, 403-424.

[2] Barbour, A. D. and Chryssaphinou, O. (2001). Compound Poisson approximation: a user's guide. Ann. Appl. Probab. 11, 964-1002.

[3] Basrak, B. AND TAFro, A. (2014). Extremes of moving averages and moving maxima on a regular lattice. Probab. Math. Statist. 34, 61-79.

[4] Basrak, B. and Planinić, H. (2018). Compound Poisson approximation for random fields with application to sequence alignment. Preprint available at arXiv:1809.00723.

[5] Chernick, M. R., Hsing, T. and McCormick, W. P. (1991). Calculating the extremal index for a class of stationary sequences. Adv. Appl. Probab. 23, 835-850.

[6] Chol, H. (2002). Central limit theory and extremes of random fields. PhD Dissertation, Univ. of North Carolina, Chapel Hill. 
[7] Cline, D. (1983). Infinite series of random variables with regularly varying tails. Technical Report 83-24, Institute of Applied Mathematics and Statistics, Univ. British Columbia.

[8] Ferreira, H. ANd Pereira, L. (2008). How to compute the extremal index of stationary random fields. Statist. Probab. Lett. 78, 1301-1304.

[9] French, J. P. And Davis, R. A. (2013). The asymptotic distribution of the maxima of a Gaussian random field on a lattice. Extremes 16, 1-26.

[10] Jakubowski, A. (1991). Relative extremal index of two stationary processes. Stochastic Process. Appl. 37, 281-297.

[11] Jakubowski, A. And Rosiński, J. (1999). Local dependencies in random fields via a Bonferroni type inequality. Contemp. Math. 234, 85-95. Available also at arXiv:1709.01165.

[12] Jakubowski, A. And Soja-KukieŁa, N. (2019). Managing local dependencies in asymptotic theory for maxima of stationary random fields. Extremes 22, 293-315.

[13] Jakubowski, A. and Soja-Kukieta, N. Directional phantom distribution functions for stationary random fields. In preparation.

[14] Leadbetter, M. R. (1983). Extremes and local dependence in stationary sequences. Z. Wahrscheinlichkeitstheor. verw. Geb. 65, 291-306.

[15] Ling, C. (2019). Extremes of stationary random fields on a lattice. Extremes 22, 391-411.

[16] Newell, G. F. (1964). Asymptotic extremes for $m$-dependent random variables. Ann. Math. Statist. 35, 1322-1325.

[17] O'Brien, G. (1987). Extreme values for stationary and Markov sequences. Ann. Probab. 15, 281-291.

[18] Pereira L., Martins A. P. and Ferreira H. (2017). Clustering of high values in random fields. Extremes 20, 807-838.

[19] Turkman, K. F. (2006). A note on the extremal index for space-time processes. J. Appl. Prob. 43, 114-126.

[20] Wu, L. And Samorodnitsky, G. (2018). Regularly varying random fields. Preprint available at arXiv: 1809.04477. 